\title{
Glucocorticoid receptor is involved in the breed-dependent transcriptional regulation of mtDNA- and nuclear-encoded mitochondria genes in the liver of newborn piglets
}

\author{
Runsheng Li, Huafeng Zou, Yimin Jia and Ruqian Zhao*
}

\begin{abstract}
Background: Mitochondria, which are essential for the functionality of eukaryotic cells, are particularly important in metabolically active tissues such as liver. Different breeds of pigs demonstrate distinct metabolic profiles in the liver, yet little is known whether the expression and transcriptional regulation of mitochondrial genes differ between breeds.

Results: Here we used male newborn Large White (LW) and Erhualian (EHL) piglets to delineate the difference in hepatic mitochondrial gene regulation between breeds. The hepatic content of ATP was significantly higher $(p<0.01)$ in EHL piglets, which was associated with lower mtDNA copy number $(p<0.05)$. Most of the mtDNA-encoded genes (10 of 13), however, were more abundantly expressed in EHL compared to LW piglets. We also detected 3 differentially expressed nuclear-encoded mitochondrial genes, among which isocitrate dehydrogenase 2 (IDH2) and ATP synthase, $\mathrm{H}^{+}$transporting, mitochondrial Fo complex, subunit $\mathrm{d}$ (ATP5H) were expressed significantly lower, while adenylate kinase 1 (AK1) was significantly over expressed in EHL piglets. Compared to $L W$, the over expression of mtDNA-encoded genes in EHL was associated with significantly higher $(p<0.01)$ glucocorticoid receptor (GR) binding to the control region of mtDNA with no alterations in the methylation status. For nuclear-encoded genes, however, a negative correlation was observed between GR binding and mRNA expression of AK1 and ATP5H. Moreover, higher expression of AK1 in EHL piglets was also associated with lower cytosine methylation $(p<0.05)$ and hydroxymethylation $(p<0.05)$. In the promoter region.
\end{abstract}

Conclusions: These results indicate a role of the GR in the breed-dependent regulation of mitochondrial genes in the liver of newborn piglets.

Keywords: Glucocorticoid receptor, Transcriptional regulation, Mitochondrial DNA (mtDNA), Nuclear-encoded mitochondrial genes, Liver, Piglets

\section{Background}

Different breeds of pigs demonstrate diverse phenotypic traits and thus serve as valuable models for animal genetics research. Erhualian (EHL) is a Chinese indigenous pig breed, being known for its early sexual maturity, large litter size, and high tolerance to roughage and stress, but its contribution to the pork industry is restricted by the slow growth rate and high adiposity [1].

\footnotetext{
* Correspondence: zhao.ruqian@gmail.com

Key Laboratory of Animal Physiology \& Biochemistry, Ministry of Agriculture, Nanjing Agricultural University, Nanjing, Jiangsu, China
}

In contrast, Large White (LW) pigs are selected for commercial interests such as growth rate and lean meat production. Numerous studies have been undertaken to reveal the genetic and physiological mechanisms underlying the striking breed differences [2,3]. The disparity in the regulation of energy homeostasis appears to be the main attribute for the various breed-specific traits $[4,5]$.

Mitochondria play a critical role in energy homeostasis through regulating cellular fuel oxidation and ATP production [6]. Liver is one of the most metabolically active organs and serves as the centre of metabolism for all the 
major nutrients, including carbohydrates, lipids and proteins. Hepatocytes contain a large number of mitochondria which play an important role in the energy homeostasis [7]. We identified, by comparing the hepatic transcriptome of LW and EHL piglets, that mitochondrial related genes consist of $5 \%(48 / 808)$ of all the differentially expressed genes [5]. Among these mitochondrial related genes, isocitrate dehydrogenase 2 (IDH2), adenylate kinase $1(A K 1)$ and ATP synthase, $\mathrm{H}^{+}$transporting, mitochondrial Fo complex, subunit d $(A T P 5 H)$ are nuclear-encoded and closely related to cellular ATP content.

In mammals, 13 oxidative phosphorylation (OXPHOS) related proteins are encoded by mitochondria's own genome (mtDNA). The mtDNA-encoded genes, however, usually lack 3'UTR sequences, and thus are absent in the dataset of microarray which only contains probe sets generated from 3'UTR sequences [GSE33523]. It remains to be clarified whether hepatic mtDNA-encoded genes are expressed differently between the two pig breeds.

Glucocorticoid (cortisol in pigs) is known to play critical roles in energy homeostasis [8]. Intriguingly, EHL pigs have 2-3 fold higher blood cortisol level compared to LW pigs, and glucocorticoid receptor (GR, NR3C1) is expressed in breed-specific patterns in hippocampus [9], muscle [10], fat [11], and liver [12]. It has been shown that the GR, besides its classic role as a nuclear receptor, can be imported into mitochondria and binds to the glucocorticoid response elements (GREs) on the control region of mtDNA [13,14]. Nevertheless, the role of GR in the regulation of nuclear- and mtDNA-encoded mitochondrial genes in different breeds of pigs remains elusive.

The overall level of DNA methylation in the liver is reported to differ between LW and EHL piglets [15]. The role of DNA methylation in transcriptional regulation has been well demonstrated in nuclear-encoded genes, including mitochondria related genes [16]. Recently, DNA methyltransferase 1 is found to translocate to the mitochondria and both 5-methylcytosine $(5 \mathrm{mC})$ and 5 -hydroxymethylcytosine $(5 \mathrm{hmC})$ are reported present in mammalian mtDNA [17]. It is unclear, however, whether cytosine methylation and hydroxymethylation status on the promoter of the nuclear- or the control region of the mtDNA-encoded mitochondrial genes in the liver would differ between LW and EHL piglets.

Therefore, we have used male newborn piglets of LW and EHL to delineate the difference in hepatic mitochondria gene expression between pig breeds. The hepatic ATP content and mitochondrial copy numbers were detected, together with the expression of all the $13 \mathrm{mtDNA}$-encoded genes and the 3 differentially expressed nuclear-encoded mitochondrial genes. The impact of GR binding, DNA methylation and hydroxymethylation on these genes were evaluated by ChIP (chromatin immunoprecipitation) and MeDIP (methylated DNA immunoprecipitation) analyses.

\section{Methods}

\section{Animal sampling}

The newborn piglets were obtained from two neighboring pig breeding farms and sacrificed immediately after birth by exsanguination. The experiment protocol was approved by the Animal Ethics Committee at Nanjing Agricultural University, China. The slaughter and sampling procedures complied with the "Guidelines on Ethical Treatment of Experimental Animals" (2006) No. 398 set by the Ministry of Science and Technology, China and "the Regulation regarding the Management and Treatment of Experimental Animals" (2008) No.45 set by the Jiangsu Provincial People's Government. Six newborn male piglets from three litters (2 from each litter) of each purebred EHL and LW sows were sacrificed. Liver samples were immersed in liquid nitrogen immediately after collection and then stored at $-70^{\circ} \mathrm{C}$.

\section{Hepatic ATP content and relative mtDNA copy number detection}

The hepatic ATP content was measured with ENLITEN ${ }^{\circ}$ ATP Assay System (FF2000, Promega) following the manufacturer's instructions.

Total genomic DNA was isolated from liver samples and the relative mtDNA copy number was determined using real-time PCR as previously described with some modifications [18]. Real-time PCR was performed in Mx3000P (Stratagene, USA) with SYBR $^{\circledR}$ Premix Ex $\mathrm{Taq}^{\mathrm{TM}}$ II (TaKaRa, Japan) according to the manufacturer's instructions. Two microliter of diluted genomic DNA $(10 \mathrm{ng} / \mu \mathrm{l})$ was used, together with $1 \mu \mathrm{l}$ of forward primer $(10 \mu \mathrm{M})$ and reverse primer $(10 \mu \mathrm{M})$, the total volume of PCR was $25 \mu \mathrm{l}$. Primers specific for the control region of mitochondrial DNA were used for the quantification of the mtDNA molecules, whereas primers specific for the promoter region of a nuclear gene ACTB were used for standardization (Table 1). The specificity of amplification was determined by dissociation curve analysis and PCR product sequencing. Relative mtDNA copy number was calculated with the $2^{-\Delta \Delta \mathrm{Ct}}$ method [19].

\section{RNA isolation and mRNA quantification}

Total RNA was isolated from liver using the Trizol reagent (Invitrogen, USA), according to the manufacturer's instructions. Concentration of the extracted RNA was measured using NanoDrop ND-1000 Spectrophotometer [20]. RNA integrity was confirmed by denaturing agarose electrophoresis. To assess the DNA contamination, the real-time PCR was carried out using $2 \mu$ liluted RNA (about $1 \mathrm{ng} / \mu \mathrm{l}$ ) as template with the primers of 18S. Samples with $\mathrm{Ct}$ values larger than 35 were consider not contaminated by genomic DNA. M-MLV reverse transcriptase (Promega, USA) and dN6 random primer (Takara, Japan) were used to synthesize cDNA 
Table 1 Primer sequences

\begin{tabular}{|c|c|c|c|c|}
\hline Primer name & Sequence & Source sequence & $\operatorname{Tm}\left({ }^{\circ} \mathrm{C}\right)$ & Used for \\
\hline \multirow[t]{2}{*}{$\operatorname{cox} 1$} & F: TGGTGCCTGAGCAGGAATAGTG & ENSSSCG00000018075 & 64 & mRNA quantification \\
\hline & R: ATCATCGCCAAGTAGGGTTCCG & & & \\
\hline \multirow[t]{2}{*}{$\operatorname{cox} 2$} & F: GCTTCCAAGACGCCACTTCAC & ENSSSCG00000018078 & 64 & mRNA quantification \\
\hline & R: TGGGCATCCATTGTGCTAGTGT & & & \\
\hline \multirow[t]{2}{*}{$\operatorname{cox} 3$} & F: GGCTACAGGGTTTCACGGGTTG & ENSSSCG00000018082 & 64 & mRNA quantification \\
\hline & R: TCAGTATCAGGCTGCGGCTTCA & & & \\
\hline \multirow[t]{2}{*}{ ND3 } & F: AGCACGCCTCCCATTCTCAAT & ENSSSCG00000018084 & 64 & mRNA quantification \\
\hline & R: TGCTAGGCTTGCTGCTAGTAGG & & & \\
\hline \multirow[t]{2}{*}{ CYTB } & F: CTGAGGAGCTACGGTCATCACA & ENSSSCG00000018094 & 64 & mRNA quantification \\
\hline & R: GCTGCGAGGGCGGTAATGAT & & & \\
\hline \multirow[t]{2}{*}{ ND1 } & F: TCCTACTGGCCGTAGCATTCCT & ENSSSCG00000018065 & 64 & mRNA quantification \\
\hline & R: TTGAGGATGTGGCTGGTCGTAG & & & \\
\hline \multirow[t]{2}{*}{ ND2 } & F: ATCGGAGGGTGAGGAGGGCTAA & ENSSSCG00000018069 & 64 & mRNA quantification \\
\hline & R: GTTGTGGTTGCTGAGCTGTGGA & & & \\
\hline \multirow[t]{2}{*}{ ND4L } & F: GATCGCCCTTGCAGGGTTACTT & ENSSSCG00000018086 & 64 & mRNA quantification \\
\hline & R: CTAGTGCAGCTTCGCAGGCT & & & \\
\hline \multirow[t]{2}{*}{ ND4 } & F: TCGCCTATTCATCAGTAAGTCA & ENSSSCG00000018087 & 64 & mRNA quantification \\
\hline & R: GGATTATGGTTCGGCTGTGTA & & & \\
\hline \multirow[t]{2}{*}{ ND5 } & F: CGGATGAGAAGGCGTAGGAA & ENSSSCG00000018091 & 64 & mRNA quantification \\
\hline & R: GCGGTTGTATAGGATTGCTTGT & & & \\
\hline \multirow[t]{2}{*}{ ND6 } & F: ACTGCTATGGCTACTGAGATGT & ENSSSCG00000018092 & 64 & mRNA quantification \\
\hline & R: CTTCCTCTTCCTTCAACGCATA & & & \\
\hline \multirow[t]{2}{*}{ ATP6 } & F: ACTCATTCACACCCACCACACA & ENSSSCG00000018081 & 64 & mRNA quantification \\
\hline & R: CCTGCTGTAATGTTGGCTGTCA & & & \\
\hline \multirow[t]{2}{*}{ ATP8 } & F: TGCCACAACTAGATACATCC & ENSSSCG00000018080 & 62 & mRNA quantification \\
\hline & R: GCTTGCTGGGTATGAGTAG & & & \\
\hline \multirow[t]{2}{*}{$\mathrm{IDH} 2$} & F: GGACAGTCACCCGCCACTA & ENSSSCG00000001852 & 62 & mRNA quantification \\
\hline & R: CGTCCAGGCAAAGATGCTG & & & \\
\hline \multirow[t]{2}{*}{$A K 1$} & F: TTGGACATGCTCCGAGACGC & ENSSSCG00000005627 & 62 & mRNA quantification \\
\hline & R: CCGATCTTCCGCTCAAACTCTT & & & \\
\hline \multirow[t]{2}{*}{ ATP5H } & F: CTACCTGAGAAGCCACCTGC & NM_001244684 & 62 & mRNA quantification \\
\hline & R: GCTGCCCACCTATGACCAC & & & \\
\hline \multirow[t]{2}{*}{ PPIA } & F: GACTGAGTGGTTGGATGG & ENSSSCG00000016737 & 62 & mRNA quantification \\
\hline & R: TGATCTTCTTGCTGGTCTT & & & \\
\hline \multirow[t]{2}{*}{185} & F: CCCACGGAATCGAGAAAGAG & ENSSSCG00000001502 & 64 & DNA contamination detection \\
\hline & R: TTGACGGAAGGGCACCA & & & \\
\hline \multirow[t]{2}{*}{ MT_controlregion } & F: CCCTATAACGCCTTGCCAAACC & & 62 & ChIP, MeDIP, mtDNA copy number \\
\hline & R: GGGTAGGTGCCTGCTITCGTAG & & & \\
\hline \multirow[t]{2}{*}{ MT_GRE_neg } & F: TCGCCTATTCATCAGTAAGT & & 62 & ChIP \\
\hline & R: GAGGATGTTAGTCCGTGGG & & & \\
\hline \multirow[t]{2}{*}{ MT_MeDIP_neg } & F: CTCAGTAGCCATAGCAGTA & & 62 & MeDIP \\
\hline & R: TGGACTTGGGTTGATTGT & & & \\
\hline IDH2_promoter & F: GGTTCAACAAGCCTTACTCACA & & 62 & ChIP, MeDIP \\
\hline
\end{tabular}


Table 1 Primer sequences (Continued)

\begin{tabular}{|c|c|c|c|}
\hline & R: GGAGACAGGAGCCGCATAG & & \\
\hline \multirow[t]{2}{*}{ AK1_promoter } & F: TGGGAGATGGATATGTGGGC & 62 & ChIP, MeDIP \\
\hline & R: GCATGGATTCTGGGGACTGT & & \\
\hline \multirow[t]{2}{*}{ ATP5H_promoter } & F: CCAAAGGTACATGGACTGC & 62 & ChIP, MeDIP \\
\hline & R: GCATCTTGGGTGGTTITAT & & \\
\hline \multirow[t]{2}{*}{ PPIA_GRE_neg } & F: GTTCACAGGGTGGTGACTT & 62 & ChIP \\
\hline & R: CAGGACCCGTATGCTTCA & & \\
\hline \multirow[t]{2}{*}{ ACTB_MeDIP_neg } & F: CTGGGCATCAGAACCTGT & 62 & MeDIP, mtDNA copy number \\
\hline & R: GAGCAATCCCCTGAAGAA & & \\
\hline
\end{tabular}

from $2 \mu \mathrm{g}$ of total RNA from each sample according to the manufacturer's instructions, the final cDNA volume of reverse transcription was $25 \mu \mathrm{l}$. After that, $2 \mu \mathrm{l}$ of diluted cDNA (1:100) was used for the real-time PCR detection. Several reference genes (18S, PLC4, GAPDH, $A C T B$ and PPIA) were tested for mRNA quantification. The expression stability was evaluated by geNorm [21] and peptidylprolyl isomerase A (PPIA) was shown to be most the stable. Additionally, PPIA seems to be stable between indigenous Chinese pig breeds and western breeds [22]. Therefore PPIA was used as the reference gene. The real-time PCR procedure in this section was similar regarding mtDNA copy number detection, except the template. Relative mRNA expression was calculated with the $2^{-\Delta \Delta C t}$ method. All primers used for this experiment were listed in Table 1.

\section{Chromatin immunoprecipitation (ChIP)}

ChIP analysis was performed as previously described with some modifications [14,23]. Briefly, $200 \mathrm{mg}$ of frozen liver samples were ground in liquid nitrogen and washed with phosphate-buffered saline (PBS) containing protease inhibitor cocktail (Roche, USA). After crosslinking in $1 \%$ formaldehyde, the reaction was stopped with $2.5 \mathrm{M}$ glycine. The pellets were washed with PBS and lysed with SDS lysis buffer $(50 \mathrm{mM}$ Tris- $\mathrm{HCl}$ pH 8.0, 10 mM EDTA, 1\% SDS) containing protease inhibitors. Cross-linked samples were sonicated for $10 \mathrm{~min}$ on ice with 10-s on/off intervals (Sonics Vibra, USA). The samples were then centrifuged at $12,000 \mathrm{rpm}$ for $10 \mathrm{~min}$ at $4^{\circ} \mathrm{C}$ to remove cell debris from the crude chromatin preparations. The average length of sonicated chromatin was around 200 to $500 \mathrm{bp}$, determined by resolving it on a $1 \%$ agarose gel. After pre-clearance of the resulting chromatin with pre-cleared Protein A/G PLUS-Agarose (sc-2003, Santa Cruz), the immunoprecipitation was performed with $2 \mu \mathrm{g}$ of a specific GR antibody (sc-1004x, Santa Cruz) overnight at $4^{\circ} \mathrm{C}$. DNA fragments were released by reverse cross-linking from the immunoprecipitated complex at $65^{\circ} \mathrm{C}$ for $5 \mathrm{~h}$. Thereafter the DNA fragments were treated with Proteinase $\mathrm{K}$
(Sunshine, China) at $45^{\circ} \mathrm{C}$ for $1 \mathrm{~h}$. After phenol/chloroform extraction, the DNA fragments were precipitated by ethanol. Finally, the samples were resuspended in $100 \mu \mathrm{l}$ Tris buffer (10 mM Tris, $\mathrm{pH} 8.5)$ and $2 \mu \mathrm{l}$ of the immunoprecipitated DNA were used as a template for the real-time PCR detection.

The primers designed for the coding region of PPIA with absence of the GRE were used as the negative control for the enrichment of GR binding on the promoter of nuclear encoded genes. For mtDNA, primers designed for the mtDNA region without any putative GRE were used as the negative control. The negative control primers are listed in Table 1. The real-time PCR procedure in this section was similar to that used for mtDNA copy number detection, except the template. The relative GR binding was calculated with the $2^{-\Delta \Delta C t}$ method, using the negative control primer as a reference.

\section{$5 \mathrm{mC}$ and $5 \mathrm{hmC}$ Immunoprecipitation}

MeDIP analysis was performed as previously described [24] with some modifications. Total genome DNA was sonicated (10 min on ice with $10 \mathrm{~s}$ on/off intervals) to yield DNA fragments of 200 to $500 \mathrm{bp}$ in length (Sonics Vibra, USA). One microgram of fragmented DNA was heat denatured to produce single-stranded DNA and immunoprecipitation was performed overnight at $4^{\circ} \mathrm{C}$ with $1 \mu \mathrm{g}$ anti-5mC antibody (ab10805, Abcam), or anti5 hmC antibody (39999, Active Motif). Pre-cleared Protein A/G PLUS-Agarose (sc-2003, Santa Cruz) was used to immunoprecipitate the antibody/DNA complex. The beads bound with immune complexes were washed to remove nonspecific binding and resuspended in $250 \mu \mathrm{l}$ of digestion buffer containing proteinase K. Finally, the MeDIP DNA was purified with phenol/chloroform extracting and then ethanol precipitated. The resulting DNA fragments were then resuspended in $100 \mu \mathrm{l}$ Tris buffer $(10 \mathrm{mM}$ Tris, $\mathrm{pH} 8.5)$ and $2 \mu \mathrm{l}$ of the DNA was used for the real-time PCR detection.

The primers designed for the $A C T B$ promoter region without the $\mathrm{CpG}$ site were used as a negative control. For mtDNA, the primers designed for the mtDNA 
region without $\mathrm{CpG}$ site were used. All primers are listed in Table 1. The real-time PCR procedure in this section was similar to that used for mtDNA copy number detection except the template. The relative methylation status was calculated using the $2^{-\Delta \Delta C t}$ method. The negative control primer was used as the reference.

\section{Bioinformatics approach and statistical analysis}

The 5 ' flanking sequence of all the nuclear genes investigated was fetched from Ensembl (Sscrofa10.2, Ensembl release 69). The $5000 \mathrm{bp}$ of the $5^{\prime}$ flanking sequence of the nuclear-encoded gene was used to predict CpG islands and GREs. The control region of the mitochondrion was defined as in previous reports $[14,25]$.

The CpG islands on the promoters of the candidate genes were assessed by Methyl Primer Express v1.0 (Applied Biosystems, USA) using the following criteria: \%GC > 50\%, length $>200 \mathrm{bp}, \mathrm{CpG}$ observed/CpG expected $>0.6$. The putative GREs in the promoter region were predicted by Transcription Element Search System (TESS, http://www. cbil.upenn.edu/cgi-bin/tess/tess). The promoter region containing multiple GREs was used to design primers for GR binding detection. The promoter region with a high density of GREs, inside the CpG islands was used to design the primer for the GR binding and methylation status detection.

To access the correlations of ATP content, mtDNA copy number, gene expression, DNA (hydroxy)methylation status and GR binding status in all 12 samples $(6 \mathrm{LW}$ and 6 EHL), a cluster analysis was carried out using all previously listed characteristics. Each characteristic value was $\log 2$ transformed for the cluster analysis. The hierarchical cluster analysis was preformed with $\mathrm{MeV}$ software (version 4.2.6). Correlation (centred) similarity matrix and average linkage algorithms were used in the cluster analysis.

All data are presented as the mean \pm SEM and were analyzed using the $t$ test for independent-samples with
SPSS 17.0 for windows. Differences were considered significant when $\mathrm{p}<0.05$.

\section{Results}

Hepatic ATP content and mtDNA copy number in newborn piglets differ between breeds

The hepatic ATP content was almost 5 fold higher $(\mathrm{p}<0.01$, Figure $1 \mathrm{~A})$ in EHL than that in LW piglets (6.3 vs. $1.3 \mu \mathrm{g} / \mathrm{g})$. The copy number of mtDNA in the liver, however, was significantly lower in EHL than in LW $(\mathrm{p}<0.05$, Figure $1 \mathrm{~B})$.

\section{The expression of mtDNA-encoded genes and nuclear-encoded mitochondrial genes}

In contrast to the mtDNA copy number, most of the mtDNA-encoded genes (10 of 13) showed a higher expression in EHL than that in LW (Figure 2A). COX1, COX2, ND2, ND3, ND4, CYTB and ND4L were significantly up-regulated in the liver of EHL piglets compared to LW ( $\mathrm{p}<0.05)$, while ATP6, ND1, ND5 tended to be higher $(\mathrm{p}<0.1)$. For the three nuclear-encoded mitochondrial genes investigated, $I D H 2$ and $A T P 5 H$ were significantly down-regulated, while $A K 1$ was significantly up-regulated in EHL when compared to LW (Figure 2B).

\section{GR binding and methylation status of the control region of mtDNA}

The control region of the mtDNA spans from 15436 to 16770 bp containing a CpG island (from 15856 to $16655 \mathrm{bp}$ ). Two putative GR binding sites were predicted in this region (Figure 3A). GR binding to the control region of mtDNA was significantly higher $(\mathrm{P}<0.01)$ in $\mathrm{EHL}$ than that in LW (Figure 3B), whereas the methylation status of this region did not show any breed difference (Figure 3C).
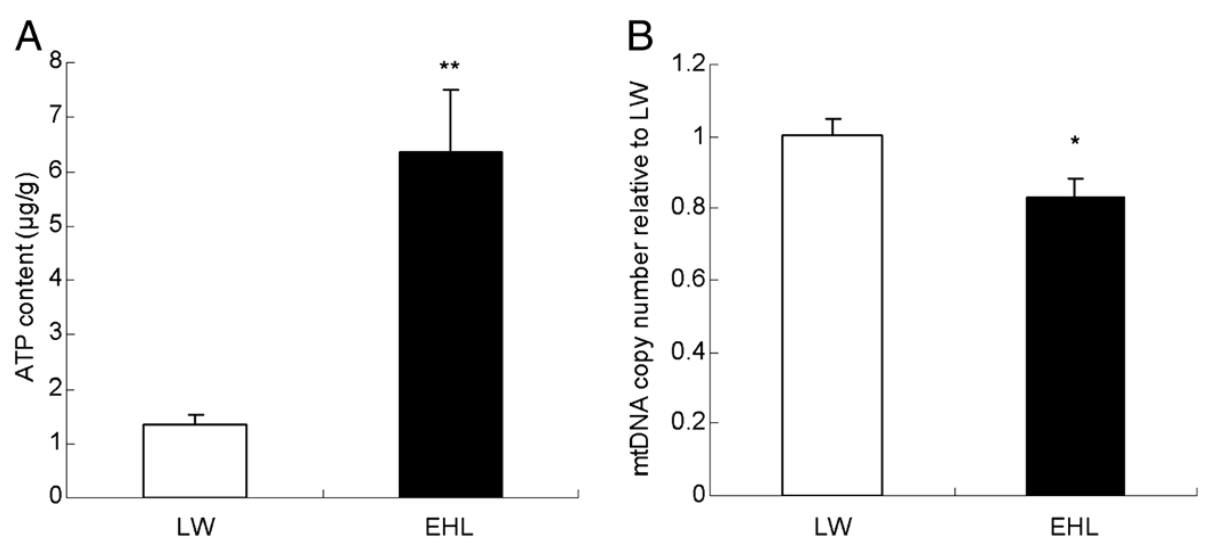

Figure 1 Hepatic ATP content (A) and the relative mtDNA copy number (B) in newborn EHL and LW piglets. Values are mean \pm SEM, $n=6 /$ breed. * means $p<0.05$, ** means $p<0.01$. 

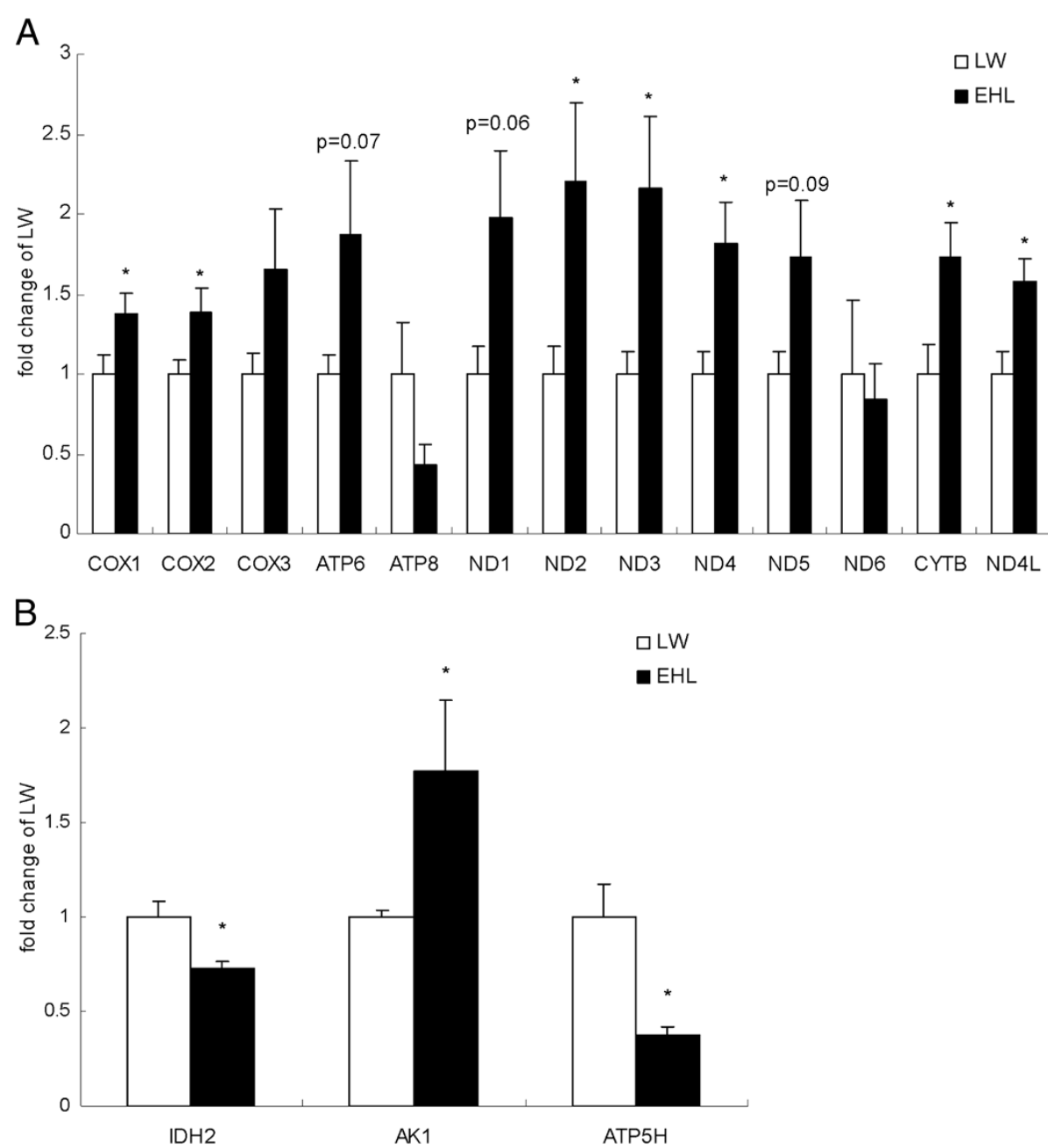

Figure 2 Hepatic expression of mtDNA- (A) and nuclear-encoded (B) mitochondrial genes in EHL and LW pigs. Values are mean \pm SEM, $n=6 /$ breed. ${ }^{*}$ means $p<0.05$, $p$ values between 0.05 and 0.1 are marked above the bar.

\section{GR binding and methylation status of promoter of nuclear-encoded mitochondrial genes}

Figure 4 shows the promoter regions of $I D H 2$ (Figure 4A), $A K 1$ (Figure 4D) and ATP5H (Figure 4G). Both $I D H 2$ and $A K 1$ have a $\mathrm{CpG}$ island in their promoter region. There are 10 and 29 putative GREs in the CpG island of $I D H 2$ and $A K 1$, respectively. Since there was a sequence gap located in the proximal $5^{\prime}$ flanking sequence of porcine $A T P 5 H$ gene, we used the sequence of 1715 bp prior to the ATG site for further assessment. No CpG islands were found within the sequence, but 15 putative GREs were observed.

Neither GR binding nor the methylation status of the $I D H 2$ promoter region showed differences between the two pig breeds (Figure 4B, C). GR binding to the $A K 1$ promoter region, however, was significantly lower in EHL, associated with lower levels of cytosine methylation $(\mathrm{p}<0.05)$ and hydroxymethylation $(\mathrm{p}<0.05)$, compared to LW piglets (Figure 4E, F). In contrast, GR binding to the promoter region of $A T P 5 H$ was significantly higher $(\mathrm{p}<0.05)$ in EHL compared to LW, while the methylation and hydroxymethylation status did not differ between breeds (Figure 4H, I).

\section{The hierarchical cluster analysis of all characteristics}

By the cluster analysis for samples, the EHL and LW piglets can be divided into two clusters. Piglets from the same sow (EHL1 and EHL 2, EHL 3 and EHL 4, EHL 5 and EHL 6, LW3 and LW4) seem to have more similar pattern. In the cluster analysis for characteristics, the 11 of 13 (except ND6 and ATP8) mtDNA-encoded genes make up the largest cluster, all 11 genes showed very similar expression patterns. The ATP content was mostly related to $A K 1$ expression and the GR binding to the mtDNA. The expressions of $I D H 2$ and ATP5H were clustered into the same one. GR binding and (hydroxy) methylation status of the $A K 1$ promoter were highly correlated (Figure 5). 

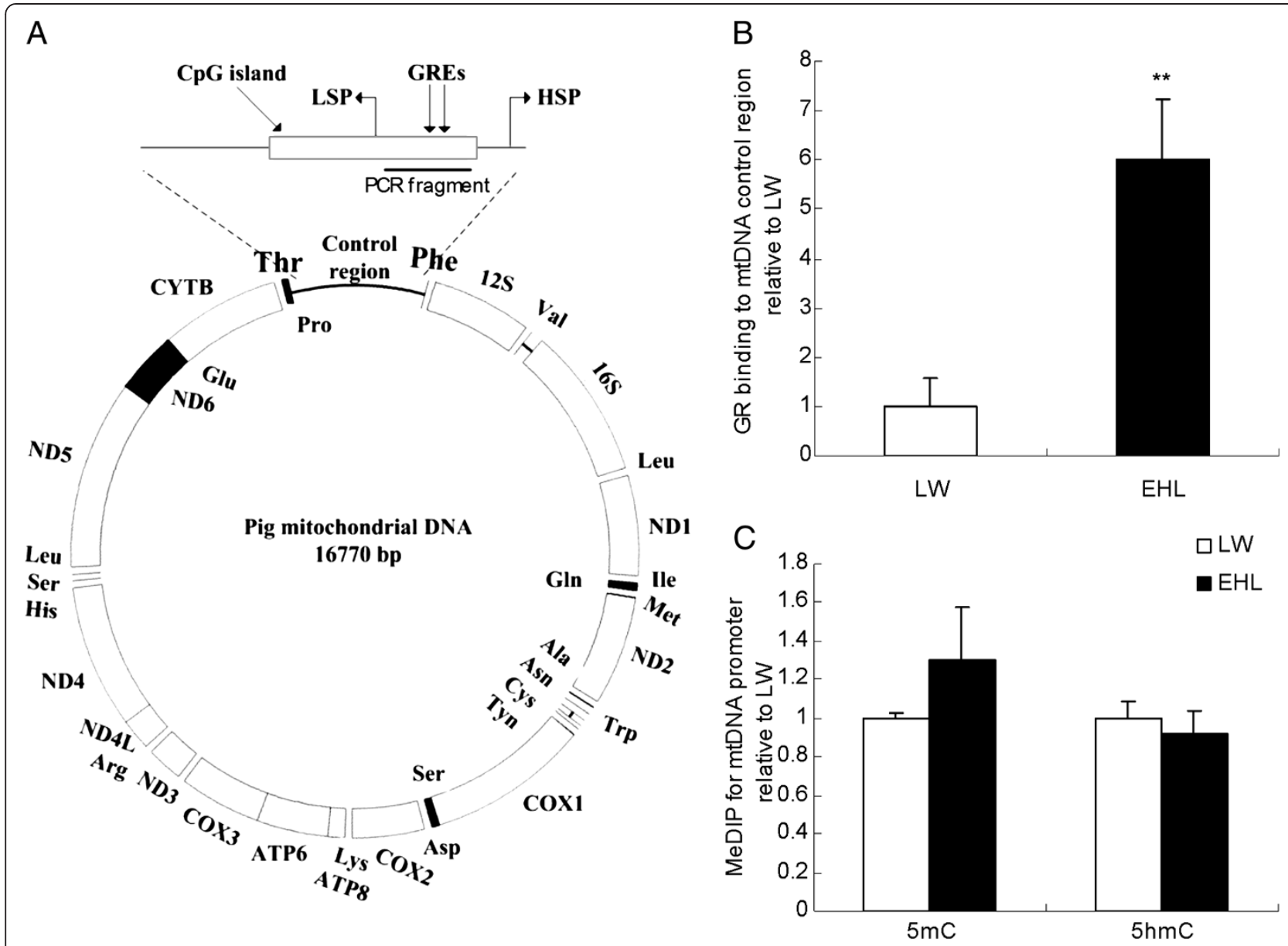

Figure 3 Schematic structure of pig mtDNA (A), GR binding (B), and DNA (hydroxyl)methylation status of CpG island on the control region of $\mathrm{mtDNA}(\mathrm{C})$. Values are mean $\pm \mathrm{SEM}, \mathrm{n}=6 /$ breed. ${ }^{*}$ means $p<0.01$ between breeds; GREs, glucocorticoid response elements; HSP, heavy strand promoter; LSP, light strand promoter.

\section{Discussion}

Like other mammals, the pig contains a mtDNA genome which encodes 13 proteins, along with $12 \mathrm{~s}, 16 \mathrm{~s}$ rRNAs and 22 tRNAs. The 13 proteins synthesized on mitochondrial ribosomes are incorporated into respiratory complex I, III, IV, and V along with approximately 67-nuclear-encoded subunits [26]. Previous studies have shown breed differences in mitochondrial function in muscle and fat in the pig $[27,28]$. The transcript profiling of ESTs from the semimembranosus muscle demonstrates elevated expression of mitochondrial related enzymes (NADH dehydrogenase, cytocrome c oxidase and cytocrome b reductase) in the skeletal muscle of Piau, a naturalized Brazilian pig breed with slow growth rate, high fat deposition and favorable meat quality, compared to commercial breeds (Large White and Duroc), implicating breed differences in oxidative and glycolytic metabolism [29]. Similar breed differences were reported in Korean native pigs who demonstrate higher expression of the 3 mitochondrial proteins: succinate dehydrogenase, NADH-ubiquinone oxidoreductase, and glycerol-3-phosphate dehydrogenase in the muscle as compared with Landrace [4]. In contrast, genes involved in mitochondrial energy metabolism are reported to be more abundantly expressed in adipose tissue of LW pigs, compared to Basque pigs showing low growth rate and high fat content [30].

Previous studies indicate striking breed differences in hepatic metabolism in the pig [5,31]. Although it is well known that hepatic metabolism is closely related to mitochondrial functions, no direct evidence of breed differences in mitochondrial function is available in the pig. Here we describe, for the first time to our knowledge, breed differences in mitochondrial function in porcine liver. Most of the mtDNA-encoded genes are expressed in higher abundance in the liver of EHL piglets, indicating more active OXPHOS function which results in higher deposition of hepatic ATP. Nonetheless, mRNA expression is not directly related to the function. In some cases, the levels of mRNA and protein are inversely correlated. 


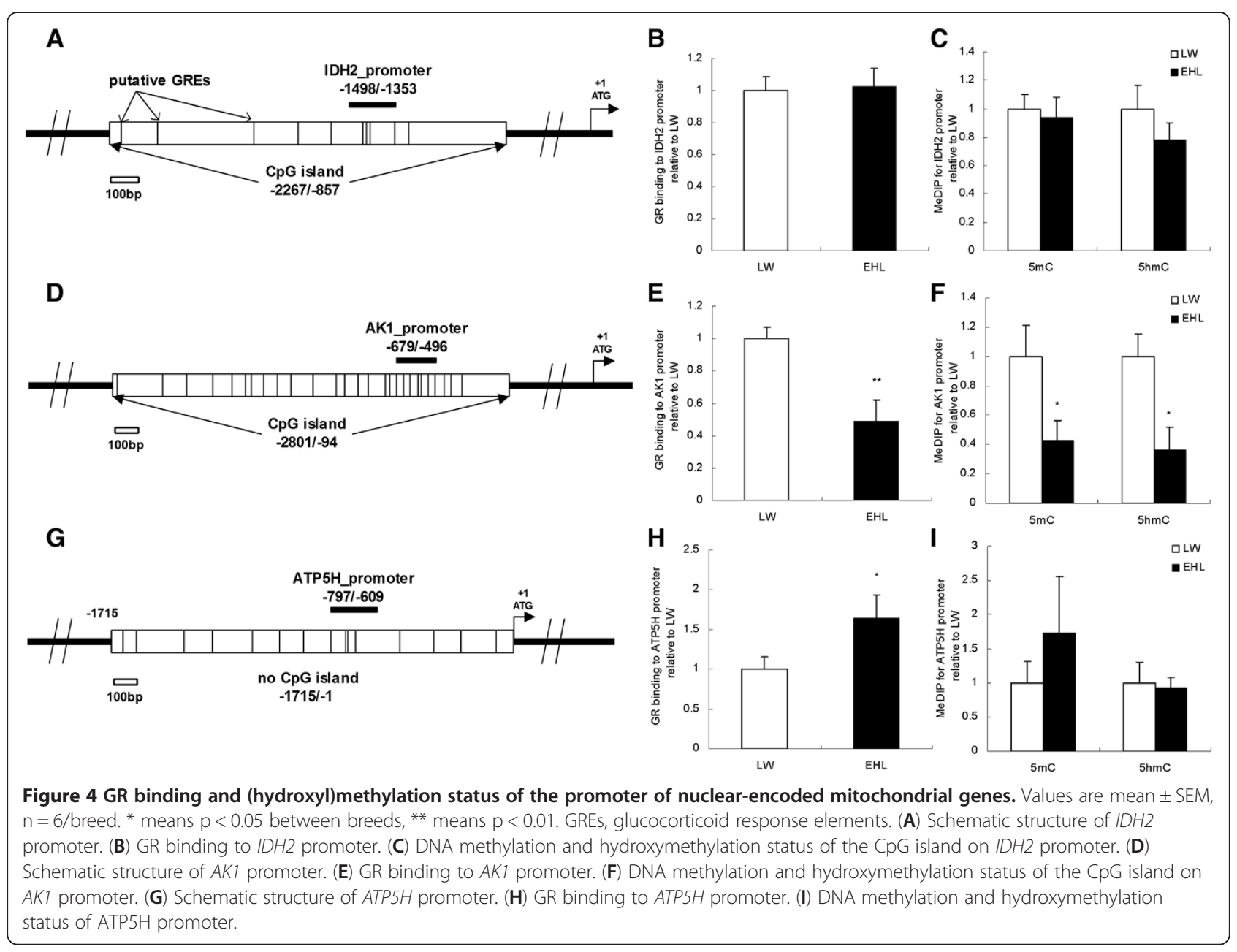

Therefore, it is possible that some OXPHOS related genes were expressed lower in EHL piglets, while the majority of the relevant genes were more highly expressed in EHL piglets, when compared with LW. Additionally, the lower IDH 2 and ATP5H gene expression in EHL could be a feed-back effect of the higher ATP content. Nevertheless, more direct evidence is still needed to support our presumption. For ATP8, we didn't observe a lower expression because of the high standard deviation in each group. Although the PCR product of ATP8 primer was confirmed by sequencing, our primer did not appear to amplify the ATP8 sequence very well. Note that there are 3 missing values of the LW group because of a bad dissociation curve (Figure 5). The expression of ND6 was also not affected by the GR. This may be explained by the ND6 being encoded by the light strain, while the other genes were encoded by the heavy strain.

The differences in mitochondrial function between breeds may contribute to some physiological properties. In our previous "Gene Ontology" analysis using hepatic differentially expressed genes between LW and EHL piglets, the "ATP binding" ranked the third in significantly enriched "molecular function" terms [5]. The higher liver ATP content in EHL may activate several transmembrane proteins that use ATP hydrolysis as an energy source for the transport of a variety of substances through cellular membranes, especially cholesterol and lipoprotein $[32,33]$. And when compared to LW, the EHL piglets showed elevated serum total cholesterol, high density lipoprotein cholesterol and low density lipoprotein cholesterol, as well as higher liver content of cholesterol [5]. In type 1 diabetes the liver mitochondrial proteome shifts to support ATP production [34]. The EHL piglets, which has lower serum insulin than LW, gets higher hepatic OXPHOS activity.

To our surprise, reduced mtDNA copy number was detected in the liver of the EHL piglets, indicating lower mitochondrial biogenesis. Improved hepatic mitochondrial function is usually associated with enhanced mitochondrial biogenesis, which sometimes corresponds with 


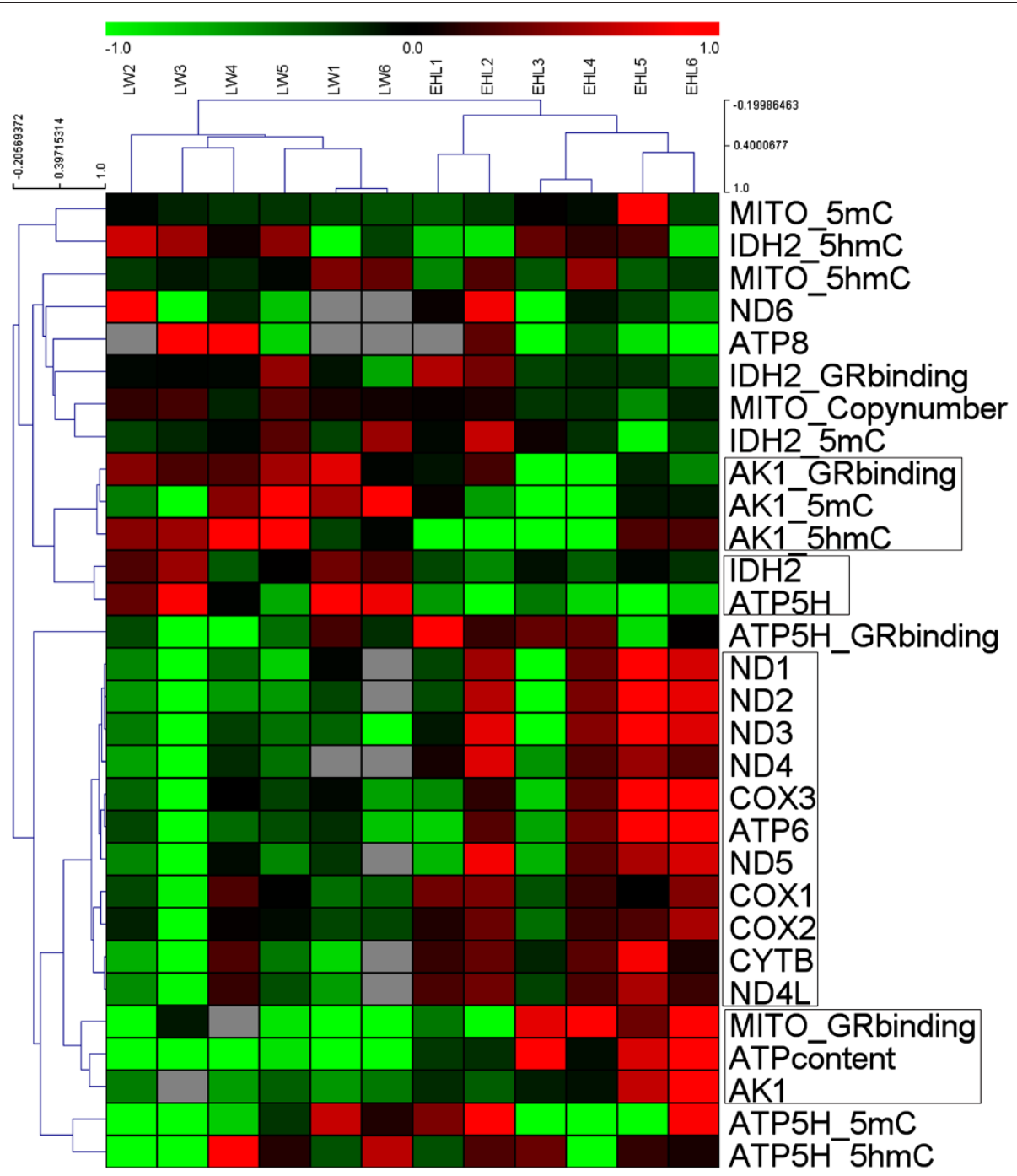

Figure 5 Hierarchical cluster analysis of the characteristics in liver from Large White (LW) and Erhualian (EHL) piglets. The figure was drawn by MeV software (version 4.2.6). Correlation (centred) similarity matrix and average linkage algorithms were used in the cluster analysis. Each row represents an individual characteristic, and each column represents a sample. The dendrogram at the left side and the top displays similarity of expression among characteristics and samples individually. The color legend represents the characteristic level, with red indicating high expression levels and green indicating low expression levels, while the gray blocks in the figure indicate missing values. The codes on the legend are log2-transformed values.

up-regulation of peroxisome proliferator-activated receptor $\gamma$ coactivator-1 $\alpha$ (PGC-1 $\alpha$, PPARGC1A) [35]. PPARGC1A was up-regulated in several liver transcriptome datasets when treated with dexamethasone [GSE24255, GSE24256 and GSE34229], but did not differ between EHL and LW piglets in our porcine liver transcriptome dataset [GSE33523]. The mtDNA copy number, however, was not always associated with mtDNA transcription and mitochondrial biogenesis. In our correlation analysis, the mtDNA copy number was even reversely related to the ATP content (data not shown). Previous research on the rat showed that an age-related decrease in mtDNA copy number was not associated with COX transcript levels in liver and red soleus muscle [36]. Increased mtDNA copy number by exogenous mitochondrial transcription factor A (TFAM) in mouse did not result in higher respiratory chain capacity or mitochondrial mass [37].

GR, as a transcription factor, can activate or repress the expression of nuclear-encoded genes, depending on the nature of the GREs. It is known that some motifs like "GGTACANNNTGTTCT" in the promoter region have been recognized as a positively modulated GRE [38], whereas the motif like "TGGACG" was suggested as a negatively modulated GRE (nGRE) $[39,40]$. The GREs predicted in the promoters of the 3 nuclearencoded mitochondrial genes are predominantly nGREs. The density of putative GRE in the promoter region may imply a probability of the GR binding to the region. The 
average GRE number in the promoter region of $I D H 2$, $A K 1$ and $A T P 5 H$ is $0.71,1.07$ and 0.88 per 100 bp, respectively (Figure 4). High density of GREs in the promoter region of a gene indicates that this gene may be more sensitive to glucocorticoids. In this study, we found that GR binding to the promoter region is inversely related to the expression of $A K 1$ and $A T P 5 H$ in LW and EHL piglets, respectively, indicating an inhibitory role of the GR in the transcriptional regulation of these two genes. Unlike nuclear encoded genes whose transcription is controlled by their respective promoters, mtDNA-encoded genes share a common control region, also known as the D-loop region [41]. Several single nucleotide polymorphisms (SNPs) have been identified in the control region of mtDNA between pig breeds [25,42]. In almost all situations, the SNPs between the pig breeds only show differences in proportion. The role of the GR in the regulation of mtDNA transcription has not been described in the pig. GRE binding sites like "CAGAGTGTACA" and "TCTTATAAAACA" were predicted in the control region of mtDNA. Glucocorticoid is reported to increase mitochondrial gene expression and OXPHOS activity by increasing the binding of GR to mtDNA [14]. In agreement with these findings, GR binding to the control region of mtDNA was 5 fold higher in the liver of EHL piglets compared to LW. By the cluster analysis, we can find that the GR binding was closely related to the expression of $11 \mathrm{mtDNA}$-encoded genes. These findings indicate complex roles of the GR in the regulation of mtDNA- and nuclear-encoded mitochondrial genes in the pig.

Although the overall level of DNA methylation in the liver was reported to be different between LW and EHL piglets [15], we failed to show breed differences in the level of cytosine methylation and hydroxymethylation in the control region of mtDNA. Among the 3 nuclearencoded mitochondrial genes investigated, only $A K 1$ is differentially modified in the promoter region, including both cytosine methylation and hydroxymethylation. The function of $5 \mathrm{hmC}$ in the nuclear genome is not yet clear. The $5 \mathrm{hmC}$ sites mainly located in the coding sequence, can alter local chromatin structure or associate with the demethylation process to induce transcription [43]. Meanwhile, the $5 \mathrm{hmC}$ located in the promoter region but not the gene body usually negatively regulates gene expression, and functions like $5 \mathrm{mC}[44,45]$. The lower levels of cytosine methylation and hydroximethylation in the promoter region of the $A K 1$ gene in the liver of EHL piglets could be associated with the higher expression levels of $A K 1$ observed in EHL piglets in comparison with LW ones. Cluster analysis showed that $5 \mathrm{mC}$ and $5 \mathrm{hmC}$ level in the promoter region of $A K 1$ were closely correlated, both of them were negatively correlated with the expression of $A K 1$ (data not shown). Further studies are needed to understand whether GR binding interacts with DNA methylation to regulate gene transcription.

\section{Conclusions}

Our results provide the first evidence for the breedspecific regulation of hepatic mitochondrial gene expression in the pig. GR is involved in the regulation of both nuclear- and mtDNA-encoded mitochondrial genes in porcine liver. The disparity in mitochondria OXPHOS function between breeds results, at least partly, from the breed-dependent role of GR in the regulation of mtDNAand nuclear- encoded mitochondrial genes in the liver of newborn LW and EHL piglets. Our findings shed new light on our understanding of the molecular mechanisms underlying breed disparities in the pig.

\section{Abbreviations}

LW: Large White; EHL: Erhualian; mtDNA: mitochondrial DNA; OXPHOS: Oxidative phosphorylation; GR: Glucocorticoid receptor; GRE: Glucocorticoid response element; $5 \mathrm{mC}$ : 5-methylcytosine; 5hmC: 5-hydroxymethylcytosine; ChIP: Chromatin immunoprecipitation; MeDIP: Methylated DNA immunoprecipitation.

\section{Competing interests}

The authors declare that they have no competing interests.

\section{Authors' contributions}

RZ designed the experiment, supervised laboratory work and critically revised the manuscript; RL mainly preformed the experiments and analyzed the data; $\mathrm{HZ}$ provided samples and carried out the ChIP experiment; YJ assisted with discussion of results and writing the manuscript. All authors read the manuscript and approved it in its final version.

\section{Acknowledgments}

This work was supported by the National Basic Research Program of China (2012CB124703), the Special Fund for Agro-scientific Research in the Public Interest (201003011), the Fundamental Research Funds for the Central Universities (KYZ200913), and the Priority Academic Program Development of Jiangsu Higher Education Institutions.

Received: 28 December 2012 Accepted: 23 April 2013 Published: 26 April 2013

\section{References}

1. Wang L, Zhang G, Lin F, Jiang B, Dong F, Liu H: Expression of the insulinlike growth factor system in skeletal muscle during embryonic and postnatal development in the first filial generation pigs from Erhualian and Yorkshire reciprocal crosses. Gen Comp Endocrinol 2011, 173(1):56-62.

2. Luetkemeier ES, Sodhi M, Schook LB, Malhi RS: Multiple Asian pig origins revealed through genomic analyses. Mol Phylogenet Evol 2010, 54(3):680-686.

3. Zhou QY, Fang MD, Huang TH, Li CC, Yu M, Zhao SH: Detection of differentially expressed genes between Erhualian and Large White placentas on day 75 and 90 of gestation. BMC Genomics 2009, 10:337.

4. Kim NK, Park HR, Lee HC, Yoon D, Son ES, Kim YS, Kim SR, Kim OH, Lee CS: Comparative studies of skeletal muscle proteome and transcriptome profilings between pig breeds. Mamm Genome 2010, 21(5-6):307-319.

5. Li R, Sun Q, Jia Y, Cong R, Ni Y, Yang X, Jiang Z, Zhao R: Coordinated miRNA/mRNA expression profiles for understanding breed-specific metabolic characters of liver between Erhualian and large white pigs. PLoS One 2012, 7(6):e38716.

6. Gibson BW: The human mitochondrial proteome: oxidative stress, protein modifications and oxidative phosphorylation. Int J Biochem Cell Biol 2005, 37(5):927-934

7. Florendo NT: Ribosome substructure in intact mouse liver cells. J Cell Biol 1969, 41(1):335-339. 
8. Solomon MB, Sakai RR, Woods SC, Foster MT: Differential effects of glucocorticoids on energy homeostasis in Syrian hamsters. Am J Physiol Endocrinol Metab 2011, 301(2):E307-E316.

9. Perreau V, Sarrieau A, Morméde P: Characterization of mineralocorticoid and glucocorticoid receptors in pigs: comparison of Meishan and Large White breeds. Life Sci 1999, 64(17):1501-1515.

10. Li X, Yang X, Shan B, Shi J, Xia D, Wegner J, Zhao R: Meat quality is associated with muscle metabolic status but not contractile myofiber type composition in premature pigs. Meat Sci 2009, 81(1):218-223.

11. Mostyn A, Sebert S, Litten JC, Perkins KS, Laws J, Symonds ME, Clarke L: Influence of porcine genotype on the abundance of thyroid hormones and leptin in sow milk and its impact on growth, metabolism and expression of key adipose tissue genes in offspring. J Endocrinol 2006, 190(3):631-639.

12. Zou H, Li R, Jia Y, Yang X, Ni Y, Cong R, Soloway PD, Zhao R: BreedDependent Transcriptional Regulation of 5'-Untranslated GR (NR3C1) Exon 1 mRNA Variants in the Liver of Newborn Piglets. PLoS One 2012, 7(7):e40432.

13. Casas F, Rochard P, Rodier A, Cassar-Malek I, Marchal-Victorion S, Wiesner RJ Cabello G, Wrutniak C: A variant form of the nuclear triiodothyronine receptor c-ErbAalpha1 plays a direct role in regulation of mitochondrial RNA synthesis. Mol Cell Biol 1999, 19(12):7913-7924.

14. Psarra AM, Sekeris CE: Glucocorticoids induce mitochondrial gene transcription in HepG2 cells: role of the mitochondrial glucocorticoid receptor. Biochim Biophys Acta 2011, 1813(10):1814-1821.

15. Zhang C, Li R, Zhao R: A semi-quantitative assay of overall DNA methylation status using Methyl-CpG binding protein (MBD1). BMC Res Notes 2012, 5:234

16. Kaneda M, Watanabe S, Hirao Y, Akagi S, Haraguchi S, Somfai T, Takeda K, Hirako M, Geshi M, Nagai T: 47 differences in mitochondrial DNA copy number and epigenetic patterns of mitochondria-related genes in cloned cows from the same donor cells. Reprod Fertil Dev 2012 , 25(1):171-171.

17. Shock LS, Thakkar PV, Peterson EJ, Moran RG, Taylor SM: DNA methyltransferase 1, cytosine methylation, and cytosine hydroxymethylation in mammalian mitochondria. Proc Natl Acad Sci U S A 2011, 108(9):3630-3635.

18. Piechota J, Szczesny R, Wolanin K, Chlebowski A, Bartnik E: Nuclear and mitochondrial genome responses in HeLa cells treated with inhibitors of mitochondrial DNA expression. Acta Biochim Pol 2006, 53(3):485-495.

19. Livak KJ, Schmittgen TD: Analysis of relative gene expression data using real-time quantitative PCR and the 2(-Delta Delta $C(T)$ ) Method. Methods 2001, 25(4):402-408

20. Desjardins PR, Conklin DS: Microvolume quantitation of nucleic acids. Curr Protoc Mol Biol 2011, Appendix 3:3J.

21. Vandesompele J, De Preter K, Pattyn F, Poppe B, Van Roy N, De Paepe A Speleman F: Accurate normalization of real-time quantitative RT-PCR data by geometric averaging of multiple internal control genes. Genome Biol 2002, 3(7):RESEARCH0034

22. Feng $X$, Xiong $Y$, Qian $H$, Lei $M, X u$ D, Ren Z: Selection of reference genes for gene expression studies in porcine skeletal muscle using SYBR green qPCR. J Biotechnol 2010, 150(3):288-293.

23. Jia Y, Cong R, Li R, Yang X, Sun Q, Parvizi N, Zhao R: Maternal low-protein diet induces gender-dependent changes in epigenetic regulation of the glucose-6-phosphatase gene in newborn piglet liver. J Nutr 2012, 142(9):1659-1665.

24. Cong R, Jia Y, Li R, Ni Y, Yang X, Sun Q, Parvizi N, Zhao R: Maternal lowprotein diet causes epigenetic deregulation of HMGCR and CYP7alpha1 in the liver of weaning piglets. J Nutr Biochem 2012, 23(12):1647-1654.

25. Qu KX, Wu GS, Gou X, Yan DW, Lian LS, Baig M, Zhang YP: Genetic differentiations between randomly and selectively bred pig populations in Yunnan, China. Dongwuxue Yanjiu 2011, 32(3):255-261.

26. Chan DC: Mitochondrial fusion and fission in mammals. Annu Rev Cell Dev Biol 2006, 22:79-99.

27. Werner C, Natter R, Schellander K, Wicke M: Mitochondrial respiratory activity in porcine longissimus muscle fibers of different pig genetics in relation to their meat quality. Meat Sci 2010, 85(1):127-133.

28. Chen C, Lin E, Cheng W, Sun H, Mersmann H, Ding S: Abundantly expressed genes in pig adipose tissue: An expressed sequence tag approach. J Anim Sci 2006, 84(10):2673-2683.
29. Nascimento CS, Peixoto JO, Verardo LL, Campos CF, Weller MM, Faria VR, Botelho ME, Martins MF, Machado MA, Silva FF, et al: Transcript profiling of expressed sequence tags from semimembranosus muscle of commercial and naturalized pig breeds. Genet Mol Res 2012, 11(3):3315-3328.

30. Vincent A, Louveau I, Gondret F, Lebret B, Damon M: Mitochondrial function, fatty acid metabolism and immune system are relevant features of pig adipose tissue development. Physiol Genomics 2012, 44(22):1116-1124.

31. Kim S-S, Kim S-R, Kim J-R, Moon J-K, Choi B-H, Lee J-W, Kim K-S, Kim T-H, Kim H-J, Lee C-K: Differences in Hepatic Gene Expression as a Major Distinguishing Factor between Korean Native Pig and Yorkshire. Biosci Biotechnol Biochem 2011, 75(3):451-458.

32. Wang N, Silver DL, Thiele C, Tall AR: ATP-binding cassette transporter A1 (ABCA1) functions as a cholesterol efflux regulatory protein. $J$ Biol Chem 2001, 276(26):23742-23747.

33. Wang N, Lan D, Chen W, Matsuura F, Tall AR: ATP-binding cassette transporters $\mathrm{G} 1$ and $\mathrm{G} 4$ mediate cellular cholesterol efflux to highdensity lipoproteins. Proc Natl Acad Sci U S A 2004, 101(26):9774-9779.

34. Johnson DT, Harris RA, French S, Aponte A, Balaban RS: Proteomic changes associated with diabetes in the BB-DP rat. Am J Physiol Endocrinol Metab 2009, 296(3):E422-E432.

35. Han D, Ybanez MD, Johnson HS, McDonald JN, Mesropyan L, Sancheti H, Martin G, Martin A, Lim AM, Dara L, et al: Dynamic Adaptation of Liver Mitochondria to Chronic Alcohol Feeding in Mice: BIOGENESIS, REMODELING, AND FUNCTIONAL ALTERATIONS. J Biol Chem 2012, 287(50):42165-42179.

36. Barazzoni R, Short KR, Nair KS: Effects of Aging on Mitochondrial DNA Copy Number and Cytochromec Oxidase Gene Expression in Rat Skeletal Muscle, Liver, and Heart. J Biol Chem 2000, 275(5):3343-3347.

37. Ekstrand MI, Falkenberg M, Rantanen A, Park CB, Gaspari M, Hultenby K, Rustin P, Gustafsson CM, Larsson NG: Mitochondrial transcription factor A regulates mtDNA copy number in mammals. Hum Mol Genet 2004, 13(9):935-944

38. Beato M, Chalepakis G, Schauer M, Slater EP: DNA regulatory elements for steroid hormones. I Steroid Biochem 1989, 32(5):737-747.

39. Govindan MV: Recruitment of cAMP-response element-binding protein and histone deacetylase has opposite effects on glucocorticoid receptor gene transcription. J Biol Chem 2010, 285(7):4489-4510.

40. Zong J, Ashraf J, Thompson EB: The promoter and first, untranslated exon of the human glucocorticoid receptor gene are GC rich but lack consensus glucocorticoid receptor element sites. Mol Cell Biol 1990, 10(10):5580-5585.

41. Parsons TJ, Muniec DS, Sullivan K, Woodyatt N, Alliston-Greiner R, Wilson MR, Berry DL, Holland KA, Weedn WW, Gill P, et al: A high observed substitution rate in the human mitochondrial DNA control region. Nat Genet 1997, 15(4):363-368.

42. Scheibye-Alsing K, Cirera S, Gilchrist MJ, Fredholm M, Gorodkin J: SNPfinding in pig mitochondrial ESTs. Anim Genet 2008, 39(2):193-195.

43. Mellén M, Ayata P, Dewell S, Kriaucionis S, Heintz N: MeCP2 binds to $5 \mathrm{hmC}$ enriched within active genes and accessible chromatin in the nervous system. Cell 2012, 151(7):1417-1430.

44. Robertson J, Robertson AB, Klungland A: The presence of 5hydroxymethylcytosine at the gene promoter and not in the gene body negatively regulates gene expression. Biochem Biophys Res Commun 2011, 411(1):40-43.

45. Xu Y, Wu F, Tan L, Kong L, Xiong L, Deng J, Barbera AJ, Zheng L, Zhang H, Huang S, et al: Genome-wide Regulation of $5 \mathrm{hmC}, 5 \mathrm{mC}$, and Gene Expression by Tet 1 Hydroxylase in Mouse Embryonic Stem Cells. Mol Cell 2011, 42(4):451-464.

doi:10.1186/1746-6148-9-87

Cite this article as: Li et al:: Glucocorticoid receptor is involved in the breed-dependent transcriptional regulation of mtDNA- and nuclearencoded mitochondria genes in the liver of newborn piglets. BMC Veterinary Research 2013 9:87. 